\title{
Weak lower semicontinuity of variational functionals with variable growth
}

\author{
Fu Yongqiang
}

Correspondence: fuyqhagd@yahoo. cn

Department of Mathematics, Harbin Institute of Technology, Harbin 150001, China

\section{Abstract}

In this paper, we establish the weak lower semicontinuity of variational functionals with variable growth in variable exponent Sobolev spaces. The weak lower semicontinuity is interesting by itself and can be applied to obtain the existence of an equilibrium solution in nonlinear elasticity.

2000 Mathematics Subject Classification: 49A45

Keywords: lower semicontinuity, variational functional, variable growth

\section{Introduction}

The main purpose of this paper is to study the weak lower semicontinuity of the functional

$$
F(u)=\int_{\Omega} f(x, u, \nabla u) d x
$$

where $\Omega$ is a bounded $C^{1}$ domain in $R^{n}$ and $f: R^{n} \times R^{m} \times R^{n m} \rightarrow R$ is a Caratheodory function satisfying variable growth conditions.

If $m=n=1$, Tonelli [1] proved that the functional $F$ is lower semicontinuity in $W^{1, \infty}$ $(a, b)$ if and only if the function $f$ is convex in the last variable. Later, several authors generalized this result, in which $x$ is allowed to belong to $R^{n}$ with $n>1$, see for example Serrin [2] and Marcellini and Sbordone [3]. On the other hand, if we allow the function $u$ to be vector-valued, i.e., $m>1$, then the convexity hypothesis turns to be sufficient but unnecessary. A suitable condition, termed quasiconvex, was introduced by Morrey [4]. Morrey showed that under strong regularity assumptions on $f, F$ is weakly lower semicontinuous in $W^{1, \infty}\left(\Omega, R^{m}\right)$ if and only if $f$ is quasiconvex in the last variable. Afterward, for $f$ satisfying so-called natural growth condition

$$
0 \leq f(x, \zeta, \xi) \leq a(x)+C\left(|\zeta|^{p}+|\xi|^{p}\right)
$$

where $p \geq 1, C \geq 0$ and $a(x) \geq 0$ are locally integrable, Acerbi and Fusco [5] proved that $F$ is weakly lower semicontinuous in $W^{1, p}\left(\Omega, R^{m}\right)$ if and only if $f$ is quasiconvex in the last variable. Later, Kalamajska [6] gave a shorter proof of the result in [5].

Since Kovacik and Rakosnik [7] first discussed variable exponent Lebesgue spaces and variable exponent Sobolev spaces, the field of variable exponent function spaces has witnessed an explosive growth in recent years and now there have been a large number of papers concerning these kinds of variable exponent spaces, see the

(C) 2011 Yongqiang; licensee Springer. This is an Open Access article distributed under the terms of the Creative Commons Attribution License (http://creativecommons.org/licenses/by/2.0), which permits unrestricted use, distribution, and reproduction in any medium, provided the original work is properly cited.

\section{SpringerOpen ${ }^{\circ}$}


monograph by Diening et al. [8] and the references therein. So we want to extend the result of Acerbi and Fusco to the case that $f$ satisfies variable growth conditions.

This paper is organized as the following: In Section 2, we present some preliminary facts; in Section 3, we discuss the weak lower semicontinuity of variational functionals with variable growth; in Section 4, we give an example to show that the result obtained in Section 3 can be applied to study the existence of an equilibrium solution in nonlinear elasticity.

\section{Preliminary}

In this section, we first recall some facts on variable exponent spaces $L^{p(x)}(\Omega)$ and $W^{k, p}$ ${ }^{(x)}(\Omega)$. For the details, see [7,9-13].

Let $\mathbf{P}(\Omega)$ be the set of all Lebesgue measurable functions $p: \Omega \rightarrow[1,+\infty]$.

$$
\begin{aligned}
& \rho_{p}(f)=\int_{\Omega \backslash \Omega_{\infty}}|f(x)|^{p(x)} d x+\inf _{\Omega_{\infty}}|f(x)|, \\
& \|f\|_{p}=\inf \left\{\lambda>0: \rho_{p}\left(\frac{f}{\lambda}\right) \leq 1\right\}
\end{aligned}
$$

where $\Omega_{\infty}=\{x \in \Omega: p(x)=\infty\}$. The variable exponent Lebesgue space $L^{p(x)}(\Omega)$ is the class of all functions $f$ such that $\rho_{p}(\lambda f)<\infty$ for some $\lambda=\lambda(f)>0 . L^{p(x)}(\Omega)$ is a Banach space endowed with the norm (2.2). $\rho_{p}(f)$ is called the modular of $f$ in $L^{p(x)}(\Omega)$.

For a given $p(x) \in \mathbf{P}(\Omega)$, we define the conjugate function $p^{\prime}(x)$ as:

$$
p^{\prime}(x)= \begin{cases}\infty, & \text { if } x \in \Omega_{1}=\{x \in \Omega: p(x)=1\} ; \\ 1, & \text { if } x \in \Omega_{\infty} ; \\ \frac{p(x)}{p(x)-1}, & \text { for other } x \in \Omega .\end{cases}
$$

Theorem 2.1.Let $p \in \mathbf{P}(\Omega)$. Then, the inequality

$$
\int_{\Omega}|f(x) g(x)| d x \leq r_{p}\|f\|_{p}\|g\|_{p^{\prime}}
$$

holds for every $f \in L^{p(x)}(\Omega)$ and $g \in L^{p^{\prime}(x)}(\Omega)$ with the constant $r_{p}$ depending on $p(x)$ and $\Omega$ only.

Theorem 2.2. The topology of the Banach space $L^{p(x)}(\Omega)$ endowed by the norm (2.2) coincides with the topology of modular convergence if and only if $p \in L^{\infty}(\Omega)$.

Theorem 2.3.The dual space to $L^{p(x)}(\Omega)$ is $L^{p^{\prime}(x)}(\Omega)$ if and only if $p \in L^{\infty}(\Omega)$. The space $L^{p(x)}(\Omega)$ is reflexive if and only if

$$
1<\inf _{\Omega} p(x) \leq \sup _{\Omega} p(x)<\infty .
$$

Next, we assume that $\Omega \subset R^{n}$ is a nonempty open set, $p \in \mathbf{P}(\Omega)$, and $k$ is a given natural number.

Given a multi-index $\alpha=\left(\alpha_{1}, \ldots, \alpha_{n}\right) \in N^{n}$, we set $|\alpha|=\alpha_{1}+\cdots+\alpha_{n}$ and $D^{\alpha}=D_{1}^{\alpha_{1}} \ldots D_{n}^{\alpha_{n}}$, where $D_{i}=\frac{\partial}{\partial x_{i}}$ is the generalized derivative operator.

The generalized Sobolev space $W^{k, p(x)}(\Omega)$ is the class of all functions $f$ on $\Omega$ such that $D^{\alpha} f \in L^{p(x)}(\Omega)$ for every multi-index $\alpha$ with $|\alpha| \leq k$ endowed with the norm 


$$
\|f\|_{k, p}=\sum_{|\alpha| \leq k}\left\|D^{\alpha} f\right\|_{p}
$$

By $W_{0}^{k, p(x)}(\Omega)$, we denote the subspace of $W^{k, p(x)}(\Omega)$ which is the closure of $C_{0}^{\infty}(\Omega)$ with respect to the norm (2.4).

Theorem 2.4. The space $W^{k, p(x)}(\Omega)$ and $W_{0}^{k, p(x)}(\Omega)$ are Banach spaces, which are reflexive if $p$ satisfies (2.3).

We denote the dual space of $W_{0}^{k, p(x)}(\Omega)$ by $W^{k, p^{\prime}(x)}(\Omega)$, then we have

Theorem 2.5.Let $p \in \mathbf{P}(\Omega) \cap L^{\infty}(\Omega)$. Then for every $G \in W^{k, p^{\prime}(x)}(\Omega)$, there exists a unique system of functions $\left\{g_{\alpha} \in L^{p^{\prime}(x)}(\Omega):|\alpha| \leq k\right\}$ such that

$$
G(f)=\sum_{|\alpha| \leq k} \int_{\Omega} D^{\alpha} f(x) g_{\alpha}(x) d x, \quad f \in W_{0}^{k, p(x)}(\Omega) .
$$

The norm of $W_{0}^{-k, p^{\prime}(x)}(\Omega)$ is defined as

$$
\|G\|_{-k, p^{\prime}}=\sup \left\{\frac{|G(f)|}{|| f \|_{k, p}}: f \in W_{0}^{k, p(x)}(\Omega)\right\} .
$$

Theorem 2.6. If $\Omega$ is a bounded domain with cone property, $p(x) \in C(\bar{\Omega})$ satisfies (1.2), and $q(x)$ is any Lebesgue measurable function defined on $\Omega$ with $p(x) \leq q(x)$ a. e. on $\bar{\Omega}$ and $\inf _{x \in \Omega}\left\{p^{*}(x)-q(x)\right\}>0$, then there is a compact embedding $W^{1, p(x)}(\Omega) \rightarrow L^{q(x)}$ $(\Omega)$.

Theorem 2.7. Let $\Omega$ be a domain with cone property. If $p: \bar{\Omega} \rightarrow R$ is Lipschitz continuous and satisfies (1.2), and $q(x) \in \mathbf{P}(\Omega)$ satisfies $p(x) \leq q(x) \leq p^{*}(x)$ a. e. on $\bar{\Omega}$, then there is a continuous embedding $W^{1, p(x)}(\Omega) \rightarrow L^{q(x)}(\Omega)$.

Theorem 2.8. If $p$ is continuous on $\bar{\Omega}$ and $u \in W_{0}^{1, p(x)}(\Omega)$, then

$$
\|u\|_{p} \leq C|| \nabla u \|_{p}
$$

where $C$ is a constant depending on $\Omega$.

Theorem 2.9. Suppose that $p$ satisfies $1 \leq p_{1} \leq p(x) \leq p_{2}<+\infty$. We have

(1) If $\|u\|_{p}>1$, then $\|u\|_{p}^{p_{1}} \leq \rho_{p}(u) \leq\|u\|_{p}^{p_{2}}$.

(2) If $\|u\|_{p}<1$, then $\|u\|_{p}^{p_{2}} \leq \rho_{p}(u) \leq\|u\|_{p}^{p_{1}}$.

Lemma 2.10. Suppose $\left\{f_{n}\right\}_{n=1}^{\infty}$ is bounded in $L^{p(x)}(\Omega)$ and $f_{n} \rightarrow f \in L^{p(x)}(\Omega)$ a. e. on $\Omega$. If $p(x)$ satisfies (1.2), then

$$
\lim _{n \rightarrow \infty} \int_{\Omega}\left|f_{n}\right|^{p(x)}-\left|f_{n}-f\right|^{p(x)} d x=\int_{\Omega}|f|^{p(x)} d x .
$$

\section{Semicontinuity of variational functionals}

Definition 3.1. A continuous function $f: R^{n m} \rightarrow R$ is said to be quasiconvex if for $\tilde{\xi} \in R^{n m}$, any open set $\Omega \subset R^{n}$ and $z \in C_{0}^{1}\left(\Omega, R^{m}\right)$ 


$$
f(\tilde{\xi}) \text { meas } \Omega \leq \int_{\Omega} f(\tilde{\xi}+\nabla z(x)) d x .
$$

This section will establish the following result:

Theorem 3.1. Let $\Omega$ be a bounded $C^{1}$ domain in $R^{n} . f: R^{n} \times R^{m} \times R^{n m} \rightarrow R$ satisfies.

(1) $f$ is a Caratheodory function, i.e., measurable with respect to $x$ and continuous with respect to $(\zeta, \xi)$;

(2) $0 \leq f(x, \zeta, \xi) \leq a(x)+C\left(|\zeta|^{p(x)}+|\xi|^{p(x)}\right)$ where $C \geq 0, a(x) \geq 0$ is locally integrable, $p(x)$ is Lipchitz continuous and satisfies $1 \leq p_{1} \leq p(x) \leq p_{2}<+\infty$.

Then $F(u)=\int_{\Omega} f(x, u, \nabla u) d x$ is weakly lower semicontinuous in $W^{1, p(x)}(\Omega)$ if and only if $f(x, \zeta, \xi)$ is quasiconvex with respect to $\xi$.

Theorem 3.1 is a generalization of the corresponding result in [5].

Definition 3.2. For $u \in C_{0}^{\infty}\left(R^{n}\right)$, we define

$$
\left(M^{*} u\right)(x)=M u(x)+\sum_{i=1}^{n}\left(M D_{i} u\right)(x)
$$

where

$$
(M u)(x)=\sup _{r>0} \frac{1}{\operatorname{meas} B_{r}(x)} \int_{B_{r}(x)}|u(y)| d y .
$$

Definition 3.3 Let $\Phi$ be a bijective transformation from a domain $\Omega \subset R^{n}$ onto a domain $G \subset R^{n}, \Psi=\Phi^{-1}$ is the inverse transformation of $\Phi$. Denote $y=\Phi(x)$ and

$$
\begin{aligned}
& y=\left(\phi_{1}(x), \ldots, \phi_{n}(x)\right), \\
& x=\left(\psi_{1}(y), \ldots, \psi_{n}(y)\right) .
\end{aligned}
$$

If $\phi_{1}, \ldots, \phi_{n} \in C^{k}(\bar{\Omega})$ and $\psi_{1}, \ldots, \psi_{n} \in C^{k}(\bar{G})$, we call $\Phi$ a $k$-smooth transformation.

For a measurable function $u$ on $\Omega$, we define a measurable function on $G$ by $A u(y)=$ $u(\Psi(y))$.

Lemma 3.1. If $\Phi: \Omega \rightarrow G$ is $k$-smooth transformation, $k \geq 1$, then $A$ is a bounded transformation from $W^{k, p(x)}(\Omega)$ onto $W^{k, p(\Psi(y))}(G)$ and the inverse transformation of $A$ is bounded as well.

Proof. We need only to show

$$
\|A u\|_{k, p(\Psi(y)), G} \leq C|| u \|_{k, p(x), \Omega}
$$

for $u \in W^{k, p(x)}(\Omega)$ where $C$ is a constant dependent on $\Phi$ only, because similarly by dealing with $A^{-1}$, we can also obtain

$$
\|A u\|_{k, p(\Psi(\gamma)), G} \geq C\|u\|_{k, p(x), \Omega} .
$$

As $C^{\infty}(\Omega)$ is dense in $W^{k, p(x)}(\Omega)$ (see [10]), for each $u \in W^{k, p(x)}(\Omega)$, there exists a sequence $\left\{u_{n}\right\} \subset C^{\infty}(\Omega)$ such that $u_{k} \rightarrow u$ in $W^{k, p(x)}(\Omega)$. For $u_{n}$, we have

$$
D^{\alpha}\left(A u_{n}\right)(\gamma)=\sum_{|\beta| \leq|\alpha|} M_{\alpha \beta}\left[A\left(D^{\beta} u_{n}\right)\right](\gamma)
$$


where $M_{\alpha \beta}$ is a polynomial of the derivatives of $\Psi$ with degrees not bigger than $|\alpha|$ and the orders of derivatives of the component of $\psi$ are not bigger than $|\beta|$. For $\phi \in C_{0}^{\infty}(\Omega)$, in the same way as [14] by (3.1) and letting $n \rightarrow \infty$, we obtain

$$
(-1)^{|\alpha|} \int_{G}\left(D^{\alpha} \phi\right)(\Phi(x))\left|\operatorname{det} \Phi^{\prime}(x)\right| d x=\sum_{|\beta| \leq|\alpha|} \int_{G} D^{\beta} u(x) M_{\alpha \beta}(\Phi(x))\left|\operatorname{det} \Phi^{\prime}(x)\right| \phi d x,
$$

this is to say,

$$
D^{\alpha}(A u)(\gamma)=\sum_{|\beta| \leq|\alpha|} M_{\alpha \beta}\left[A\left(D^{\beta} u\right)\right](\gamma)
$$

is satisfied in the weak sense.

As $\Phi$ is a 1-smooth transformation, there exist $C_{1}$ and $C_{2}>1$ such that

$$
C_{1} \leq\left|\operatorname{det} \Phi^{\prime}(x)\right| \leq C_{2}
$$

for $x \in \Omega$. Then,

$$
\begin{aligned}
& \int_{\Omega}\left(\frac{D^{\alpha}(A u)(y)}{C|| u \|_{k, p, \Omega}}\right)^{p(\Psi(y))} d y \\
\leq & \left.\left(\sum_{|\beta| \leq|\alpha|} 1\right)^{p_{2}} \max _{|\beta| \leq|\alpha|}\left(\sup _{\gamma \in G}\left|M_{\alpha \beta}(\gamma)\right|^{p_{2}}+1\right) \int_{\Omega}\left(\frac{\left(D^{\beta} u\right)(\Psi(y))}{C|| u \|_{k, p, \Omega}}\right)^{p(\Psi(y))} d y\right) \\
\leq & C_{2}\left(\sum_{|\beta| \leq|\alpha|} 1\right)^{p_{2}} \max _{|\beta| \leq|\alpha|}\left(\sup _{\gamma \in G}\left|M_{\alpha \beta}(\gamma)\right|^{p_{2}}+1\right) \max _{|\beta| \leq|\alpha|} \int_{\Omega}\left(\frac{\left(D^{\beta} u\right)(x)}{\left.C|| u\right|_{k, p, \Omega}}\right)^{p(x)} d x .
\end{aligned}
$$

Taking

$$
C=C_{2}\left(\sum_{|\beta| \leq|\alpha|} 1\right)^{p_{2}} \max _{|\beta| \leq|\alpha|}\left(\sup _{\gamma \in G}\left|M_{\alpha \beta}(\gamma)\right|^{p_{2}}+1\right),
$$

we have

$$
\|A u\|_{k, p(\Psi(y)), G} \leq C \mid\|u\|_{k, p(x), \Omega} .
$$

Definition 3.4. Let $\Omega$ be a domain in $R^{n}$. If $E$ is a linear operator from $W^{k, p(x)}(\Omega)$ onto $W^{k, p(x)}\left(R^{n}\right)$ satisfying: for each $u \in W^{k, p(x)}\left(R^{n}\right)$

1) $E u(x)=u(x)$ a. e. on $\Omega$,

2) $\|E u\|_{k, p, R^{n}} \leq C\|u\|_{k, p, \Omega w h e r e} C=C(k, p)$ is a constant,

then we call $E$ a simple $(k, p(x))$ extension operator of $\Omega$.

Lemma 3.2. Let $\Omega$ be a bounded $C^{k}$ domain. Then there exists a simple $(k, p(x))$ extension operator of $\Omega$.

Proof. First let $\Omega$ be the half space $R_{+}^{n}=\left\{x \in R^{n}: x_{n}>0\right\}$. For $u \in W^{k, p(x)}\left(R_{+}^{n}\right)$, define $E u$ and $E_{\alpha} u$ as the following 


$$
\begin{gathered}
E u(x)= \begin{cases}u(x), & x_{n}>0 ; \\
\sum_{j=1}^{k+1} \lambda_{j} u\left(x_{1}, \ldots, x_{n-1},-j x_{n}\right), & x_{n} \leq 0 .\end{cases} \\
E_{\alpha} u(x)= \begin{cases}u(x), & x_{n}>0 ; \\
\sum_{j=1}^{k+1}(-1)^{\alpha_{n}} \lambda_{j} u\left(x_{1}, \ldots, x_{n-1},-j x_{n}\right), & x_{n} \leq 0\end{cases}
\end{gathered}
$$

where the coefficients $\lambda_{1}, \ldots, \lambda_{m+1}$ is the unique solution of the linear system

$$
\sum_{j=1}^{k+1}(-j)^{l} \lambda_{j}=1, \quad l=0,1, \ldots, k .
$$

If $u \in C^{k}\left(\bar{R}_{+}^{n}\right)$, then $E u \in C^{k}\left(R^{n}\right)$ and $D^{\alpha} E u(x)=E_{\alpha} D^{\alpha} u(x)$. As

$$
\begin{aligned}
& \int_{R^{n}}\left(\frac{D^{\alpha} E u(x)}{C\|u\|_{k, p, R_{+}^{n}}}\right)^{p(x)} d x \\
= & \int_{R_{+}^{n}}\left(\frac{D^{\alpha} u(x)}{C\|u\|_{k, p, R_{+}^{n}}}\right)^{p(x)} d x+\int_{R_{-}^{n}}\left(\frac{\sum_{j=1}^{k+1}(-j)^{\alpha_{n}} \lambda_{j} D^{\alpha} u\left(x_{1}, \ldots, x_{n-1},-j x_{n}\right)}{C\|u\|_{k, p, R_{+}^{n}}}\right) d x \\
\leq & C(k, p, \alpha) \int_{R_{+}^{n}}\left(\frac{D^{\alpha} u(x)}{C\|u\|_{k, p, R_{+}^{n}}}\right)^{p(x)} d x,
\end{aligned}
$$

we have

$$
\|E u(x)\|_{k, p, R^{n}} \leq C|| u \|_{k, p, R_{+}^{n}}
$$

where $C=\max _{|\alpha| \leq k} C(k, p, \alpha)$.

Next, let $\Omega$ be a $C^{k}$ domain with bounded boundary. In the same way as [14], we can show that there exists a simple $(k, p(x))$ extension operator of $\Omega$.

Theorem 3.2. Let $\Omega$ be a bounded $C^{1}$ domain in $R^{n}$.Iff $: R^{n} \times R^{m} \times R^{n m} \rightarrow R$ satisfies:

(1) $f$ is a Caratheodory function;

(2) $f$ is quasiconvex with respect to $\xi$;

(3) $0 \leq f(x, \zeta, \xi) \leq a(x)+C\left(|\zeta|^{p(x)}+|\xi|^{p(x)}\right)$ for $x \in R^{n}, \zeta \in R^{n}, \xi \in R^{m}$,

where $C$ is a nonnegative constant, $a(x)$ is nonnegative and locally integrable, and $p$ $(x)$ is Lipchitz continuous and satisfies $1 \leq p_{1} \leq p(x) \leq p_{2}<+\infty$, then for each open subset $\Omega \subset R^{n}, F(u, \Omega)=\int_{\Omega} f(x, u, \nabla u) d x$ is weakly lower semicontinuous on $W^{1, p(x)}\left(\Omega, R^{m}\right)$.

Proof. We can consider $\Omega$ as a ball. Take $u \in W^{1, p(x)}\left(\Omega, R^{m}\right)$ and $\left\{z_{k}\right\} \subset W^{1, p(x)}(\Omega$, $\left.R^{m}\right)$ satisfying $z_{k} \rightarrow 0$ weakly in $W^{1, p(x)}\left(\Omega, R^{m}\right)$. Extracting a subsequence if necessary, we can suppose that

$$
\liminf _{k \rightarrow \infty} F\left(u+z_{k}, \Omega\right)=\lim _{k \rightarrow \infty} F\left(u+z_{k}, \Omega\right) .
$$

By Lemma 3.2, we can suppose that $z_{k}$ is defined on $R^{n}$, and $\left\|z_{k}\right\|_{1, p(x), R^{n}}$ is uniformly bounded with respect to $k$. As $C_{0}^{\infty}\left(R^{n}, R^{m}\right)$ is dense in $W^{1, p(x)}\left(R^{n}, R^{m}\right)$ (see [15]) and $F$ 
$(u, \Omega)$ is continuous with respect to the norm of $W^{1, p(x)}\left(\Omega, R^{m}\right)$, we can find $\left\{\omega_{k}\right\} \subset C_{0}^{\infty}\left(R^{n}, R^{m}\right)$ such that

$$
\begin{gathered}
\left\|\omega_{k}-z_{k}\right\|_{1, p, R^{n}}<\frac{1}{k}, \\
\left|F\left(u+\omega_{k}, \Omega\right)-F\left(u+z_{k}, \Omega\right)\right|<\frac{1}{k} .
\end{gathered}
$$

Therefore, we can further suppose that $\left\{z_{k}\right\}$ is in $C_{0}^{\infty}\left(R^{n}, R^{m}\right)$ and bounded in $W^{1, p(x)}$ $\left(R^{n}, R^{m}\right)$.

Take a continuous, monotone function $\eta: R^{+} \rightarrow R^{+}$such that $\eta(0)=0$ and for each measurable $B \subset \Omega$.

$$
\int_{B}\left[a(x)+C\left(|u(x)|^{p(x)}+|\nabla u(x)|^{p(x)}\right)\right] d x<\eta(\text { meas } B) .
$$

Fix $\varepsilon>0$, in the same way as [5] there exist a subsequence (still denote it by $\left\{z_{k}\right\}$ ), and a subset $A_{\varepsilon} \subset \Omega$ with meas $A_{\varepsilon}<\varepsilon$ and $\delta>0$ such that

$$
\int_{B}\left(M^{*} z_{k}^{(i)}\right)^{p(x)} d x<\varepsilon, \quad 1 \leq i \leq m,
$$

for all $k$ and $B \subset \Omega \backslash A_{\varepsilon}$ with meas $B<\delta$ and there exists sufficiently large $\lambda>0$ such that for all $i, k$,

$$
\operatorname{meas}\left\{x \in R^{n}:\left(M^{*} z_{k}^{(i)}\right)(x) \geq \lambda\right\}<\min (\varepsilon, \delta) .
$$

Denote

$$
\begin{gathered}
H_{i, k}^{\lambda}=\left\{x \in R^{n}:\left(M^{*} z_{k}^{(i)}\right)(x)<\lambda\right\}, \\
H_{k}^{\lambda}=\bigcap_{i=1}^{m} H_{i, k}^{\lambda} .
\end{gathered}
$$

Then

$$
\operatorname{meas}\left(\left(\Omega \backslash A_{\varepsilon}\right) \backslash H_{k}^{\lambda}\right) \leq \sum_{i=1}^{m} \operatorname{meas}\left(\left(\Omega \backslash A_{\varepsilon}\right) \backslash H_{i, k}^{\lambda}\right)<m \min \{\varepsilon, \delta\} .
$$

We can extend $z_{k}^{(i)}$ out of $H_{k}^{\lambda}$ to become a Lipschitz function $g_{k}^{(i)}$ with the Lipschitz constant not bigger than $C(n) \lambda$. As

$$
F\left(u+z_{k}, \Omega\right) \geq F\left(u+g_{k \prime}\left(\Omega \backslash A_{\varepsilon}\right) \cap H_{k}^{\lambda}\right)=F\left(u+g_{k}, \Omega \backslash A_{\varepsilon}\right)-F\left(u+g_{k \prime}\left(\Omega \backslash A_{\varepsilon}\right) \backslash H_{k}^{\lambda}\right),
$$

from condition (3), we have

$$
\begin{aligned}
& F\left(u+g_{k \prime}\left(\Omega \backslash A_{\varepsilon}\right) \backslash H_{k}^{\lambda}\right) \\
\leq & 2^{p_{2}-1}\left(\eta(m \varepsilon)+C(n, \Omega) \lambda^{p_{2}} \operatorname{meas}\left(\left(\Omega, \backslash A_{\varepsilon}\right) \backslash H_{k}^{\lambda}\right)\right) \\
\leq & 2^{p_{2}-1}\left(\eta(m \varepsilon)+C(n, \Omega) \sum_{i=1}^{m} \int_{\left(\Omega \backslash A_{\varepsilon}\right) \backslash H_{i, k}^{\lambda}}\left(M^{*} z_{k}^{(i)}\right)^{p(x)} d x\right) \\
\leq & 2^{p_{2}-1}(\eta(m \varepsilon)+m C(n, \Omega) \varepsilon) \\
= & O(\varepsilon) .
\end{aligned}
$$


Furthermore,

$$
\lim _{k \rightarrow \infty} F\left(u+z_{k}, \Omega\right) \geq F(u, \Omega)-O(\varepsilon)-\varepsilon-\eta[(m+2) \varepsilon] .
$$

By the arbitrariness of $\varepsilon$, we conclude the theorem.

Since semicontinuity in $W^{1, p(x)}(\Omega)$ implies semicontinuity in $W^{1, \infty}(\Omega)$, from Theorem 3.2 above and Theorem [II.2] in [5], it is immediate to get Theorem 3.1.

Next, we state a Corollary of Theorem 3.1.

Corollary 3.1. Let $\Omega$ be a bounded $C^{1}$ domain in $R^{n}$. If $: R^{n} \times R \times R^{n} \rightarrow R$ satisfies

(1) $f$ is measurable with respect to $x$ and continuous with respect to $(\zeta, \xi)$;

(2) $0 \leq f(x, \zeta, \xi) \leq a(x)+C\left(|\zeta|^{p(x)}+|\xi|^{p(x)}\right)$ where $a(x)$ is nonnegative and locally integrable, and $p(x)$ is Lipchitz continuous and satisfies $1 \leq p_{1} \leq p(x) \leq p_{2}<+\infty$.

Then $F(u)=\int_{\Omega} f(x, u, \nabla u)$ is weakly lower semicontinuous in $W^{1, p(x)}(\Omega)$ if and only if $f$ $(x, \zeta, \xi)$ is convex with respect to $\xi$.

It is immediate in view of the fact that in the case $m=1$, quasiconvexity is equivalent to convexity.

\section{Application}

We adopt the variational approach to prove the existence of an equilibrium solution in nonlinear elasticity. We consider only elastic materials possessing stored energy functions. In this case, the problem consists in finding the minimizer in $W_{0}^{1, p(x)}\left(\Omega, R^{m}\right)$ of the functional

$$
F(u)=\int_{\Omega} f(x, u, \nabla u) d x
$$

where $f$ satisfies variable growth conditions.

Example. Let $\Omega$ be a bounded $C^{1}$ domain in $R^{n} . f: R^{n} \times R^{m} \times R^{n m} \rightarrow R$ satisfies:

(1) $f$ is a Caratheodory function,

(2) $b(x)+c\left(|\zeta|^{p(x)}+|\xi|^{p(x)}\right) \leq f(x, \zeta, \xi) \leq a(x)+C\left(|\zeta|^{p(x)}+|\xi|^{p(x)}\right)$ where $c, C \geq 0$, a

$(x), b(x) \geq 0$ are locally integrable, $p(x)$ is Lipchitz continuous and satisfies $1<p_{1} \leq$ $p(x) \leq p_{2}<+\infty$;

(3) $f(x, \zeta, \xi)$ is quasiconvex with respect to $\xi$.

Then, the variational problem

$$
\inf \left\{F(u): u \in W_{0}^{1, p(x)}\left(\Omega, R^{m}\right)\right\}
$$

has a solution.

Proof. As $f(x, u, \nabla u) \geq 0, F(u)$ is bounded below. Because

$$
c \int_{\Omega}|u|^{p(x)}+|\nabla u|^{p(x)} d x \leq \int_{\Omega} f(x, u, \nabla u) d x-\int_{\Omega} b(x) d x,
$$


we know that $F(u)$ is coercive, i.e.,

$$
\lim _{\|u\|_{1, p(x) \rightarrow+\infty}} F(u)=+\infty .
$$

Then, there exists a minimizing sequence $\left\{u_{n}\right\} \subset W_{0}^{1, p(x)}\left(\Omega, R^{m}\right)$ such that

$$
\lim _{n \rightarrow \infty} F\left(u_{n}\right)=\inf \left\{F(u): u \in W_{0}^{1, p(x)}\left(\Omega, R^{m}\right)\right\} .
$$

As $F(u)$ is coercive, $\left\{u_{n}\right\}$ is bounded in $W_{0}^{1, p(x)}\left(\Omega, R^{m}\right)$, and further $\left\{u_{n}\right\}$ has a subsequence (still denoted by $\left\{u_{n}\right\}$ ) weakly convergent to $u \in W_{0}^{1, p(x)}\left(\Omega, R^{m}\right)$. Then, by the weak lower semicontinuity of $F(u)$, we have

$$
\begin{gathered}
F(u) \leq \liminf _{n \rightarrow \infty} F\left(u_{n}\right), \\
\text { i.e., } F(u)=\inf \left\{F(u): u \in W_{0}^{1, p(x)}\left(\Omega, R^{m}\right)\right\} .
\end{gathered}
$$

\section{Competing interests}

The author declares that he has no competing interests.

Received: 7 March 2011 Accepted: 19 July 2011 Published: 19 July 2011

\section{References}

1. Tonelli, L: La semicontinuita nel calcolo delle variazioni. Rend Circ Matem Palermo. 44, 167-249 (1920). doi:10.1007/ BF03014600

2. Serrin, J: On the definition and properties of certain variational integrals. Trans Am Math Soc. 101, 139-167 (1961). doi:10.1090/S0002-9947-1961-0138018-9

3. Marcellini, P, Sbordone, C: Semicontinuity problems in the calculus of variations. Nonlinear Anal. 4, 241-257 (1980). doi:10.1016/0362-546X(80)90052-8

4. Morrey, CB: Quasiconvexity and the semicontinuity of multiple integrals. Pacific J Math. 2, $25-53$ (1952)

5. Acerbi, E, Fusco, N: Semicontinuity problems in the calculus of variations. Arch Ration Mech Anal. 86, 125-145 (1984). doi:10.1007/BF00275731

6. Kalamajska, A: On lower semicontinuity of multiple integrals. Colloq Math. 74(1), 71-78 (1997)

7. Kovacik, O, Rakosnik, J: On spaces $L^{p(x)}$ and $W^{k, p(x)}$. Czechoslovak Math J. 41, 592-618 (1991)

8. Diening, L, Harjulehto, P, Hasto, P, Ruzicka, M: Lebesgue and Sobolev Spaces with Variable Exponents. Springer (2011)

9. Edmunds, D, Lang, J, Nekvinda, A: On $L^{p(x)}$ norms. Proc R Soc A. 1981, 219-225 (1999)

10. Edmunds, D, Rakosnik, J: Sobolev embedding with variable exponent. Stud Math. 143, 267-293 (2000)

11. Edmunds, D, Rakosnik, J: Sobolev embedding with variable exponent II. Math Nachr. 246-247, 53-67 (2002). doi:10.1002/1522-2616(200212)246:13.0.CO;2-T

12. Fan, $X$, Shen, J, Zhao, D: Sobolev embedding theorems for spaces $W^{k, p(x)}(\Omega)$. J Math Anal Appl. 262, 749-760 (2001). doi:10.1006/jmaa.2001.7618

13. Fu, YQ: The principle of concentration compactness in $L^{p(x)}$ spaces and its application. Nonlinear Anal. 71, 1876-1892 (2009). doi:10.1016/..na.2009.01.023

14. Adams, R: Sobolev Space. Acadimic Press, New York (1975)

15. Samko, S: The density of in generalized Sobolev spaces $W^{m, p(x)}\left(R^{n}\right)$. Doklady Math. 60, 382-385 (1999)

doi:10.1186/1029-242X-2011-19

Cite this article as: Yongqiang: Weak lower semicontinuity of variational functionals with variable growth. Journal of Inequalities and Applications 2011 2011:19. 